\title{
Social Cues of Future Sperm Competition Received during Development Affect Learning in Adult Male Fruit Flies, Drosophila melanogaster
}

\author{
Laurin S. McDowall • James Rouse • Steven M. Sait • \\ Amanda Bretman (iD
}

Received: 3 May 2018 /Revised: 10 April 2019/Accepted: 17 April 2019/Published online: 15 May 2019

(C) The Author(s) 2019

\begin{abstract}
The social environment provides males with information about the likelihood of reproductive competition. However, social context can be highly variable, and males must track their environment in order to alter reproductive investment appropriately. In addition to using information gained as adults to adjust reproductive strategies, males can use cues in early life to anticipate future mating competition and alter development of reproductive tissue. As responding to variable levels of competition may be cognitively challenging, and early life environments could influence neural development, cues of future competition during development could influence adult cognitive capacity. Male Drosophila melanogaster fruit flies reared with cues of future reproductive competition, high larval density or in the presence of adult males, develop larger accessory glands. We examined whether these early life conditions affect adult male learning ability. We assessed the learning ability of adults reared under different larval social conditions in a non-sexual and sexual context. We also measured gene expression in learning, memory and synapse-related genes previously found to respond to the adult social environment. The presence of adult males during development had no effect. Males from low larval densities, however, had better learning ability in the sexual-context assay and showed relatively higher gene expression compared to flies from high larval
\end{abstract}

L. S. McDowall · J. Rouse $\cdot$ S. M. Sait · A. Bretman $(\bowtie)$ School of Biology, Faculty of Biological Sciences, University of Leeds, Leeds LS2 9JT, UK

e-mail: a.j.bretman@leeds.ac.uk densities. This could suggest a trade-off between reproductive investment into accessory gland growth or an increased investment into neural plasticity at low density.

Keywords Development - social environment . cognition · Drosophila melanogaster

\section{Introduction}

The social environment is a source of information about potential mates and rivals. Males in particular are predicted to use social information to gauge the level of reproductive, or sperm competition, in the environment and allocate reproductive effort in a particular mating accordingly (Parker 1970; Parker and Pizzari 2010). Plasticity in reproductive strategies such as behavior (Bretman et al. 2011) and ejaculate traits (Wedell et al. 2002) under differing sperm competition environments has been observed in many species. Matching reproductive investment to the prevailing competitive environment may be cognitively challenging, as, for example, it can involve the integration of multiple sensory cues (Bretman et al. 2011) and require the capacity for memory (Rouse et al. 2018). In general, variable conditions are thought to stimulate increased investment in cognitive processes (Sol 2009), and social context can be highly variable, both spatially and temporally (Kasumovic et al. 2008). Indeed, it has been suggested that selection for plastic sperm competition strategies that are responsive to the number of competitors has 
influenced the evolution of quantity estimation (Shifferman 2012). Moreover, sexual selection has been shown to influence cognition in D. melanogaster, with lines kept under monogamous conditions showing decreased learning ability compared to polygamous lines (Hollis and Kawecki 2014).

Whilst an increased ability to process cues relating to the sperm competition environment may be beneficial, increasing cognition could represent distinct costs as neural tissue is energetically expensive to produce (Niven and Laughlin 2008). Furthermore, it has been suggested that there could be a trade-off in investment in costly neural versus reproductive tissues (the "expensive sexual tissue hypothesis") (Pitnick et al. 2006). For example, in echolocating bats, brain size appears to be negatively correlated with testes mass (Pitnick et al. 2006). As such, reducing investment into cognition may be advantageous when conditions are less heterogeneous (Sol 2009).

Developmental plasticity in response to conditions experienced during early life could have critical consequences for adult cognitive ability. For example, honey bees, Apis mellifera, have slowed growth in the mushroom bodies, which are olfactory learning centres of the brain, when reared in social isolation (Maleszka et al. 2009). Social isolation in young prairie voles, Microtus ochrogaster, results in reduced social discrimination in a socio-spatial task, which can be rescued by grouphousing at a later stage (Prounis et al. 2015). Further, jumping spiders, Marpissa muscosa, reared in socially enriched conditions enhance their learning ability compared to those reared in isolation (Liedtke and Schneider 2017). Social enrichment, however, may not impact on all aspects of cognition equally. For example, rat pups subject to maternal deprivation have reduced social but not spatial learning compared to socially stimulated individuals (Lévy et al. 2003). These studies suggest that early life conditions, and in particular social conditions, alter cognitive development.

The fruit fly, Drosophila melanogaster, is an established invertebrate model for learning studies (Dukas 2008). Social enrichment in adult flies induces the growth of synapses of the lateral ventral neurons, with a corresponding increase in sleep (Donlea et al. 2014), and the mushroom bodies can vary in size with changes in social context (Heisenberg et al. 1995). They are, therefore, generally socially-sensitive and are also one of the best studied examples of an animal using social cues to anticipate sperm competition environments. Manipulating the larval social conditions experienced by D. melanogaster by increasing larval density or adding adult males into the larval environment induces the growth of larger accessory glands in adult males, an anticipatory response to future reproductive competition (Bretman et al. 2016). As adults, males adjust mating duration (Bretman et al. 2009) and ejaculate content (Wigby et al. 2009). This requires detection of a combination of sensory cues (Bretman et al. 2011) and these cues alter the speed and accuracy with which males respond (Rouse and Bretman 2016; Bretman et al. 2017). Moreover, the response requires the anaesthesia sensitive memory pathway (Rouse et al. 2018). Therefore, in order to process cues relating to potential future sperm competition and produce their response, an enhanced cognitive ability may be required.

The larval social environment could influence cognition in two ways. Increased social contact during larval stages could stimulate cognition directly or in anticipation of an increased requirement to process sperm competition cues when adults. Alternatively, there could be a resource trade-off between reproduction and cognition. In order to test this, we assessed the learning ability of young adult males reared under differing larval density or with or without the presence of adult males (Bretman et al. 2016), using two established learning assays (Tully and Quinn 1985; Hollis and Kawecki 2014). The first of these tested olfactory learning in a non-social task, and the second examined learning in a sexual context by testing the ability of a male to find and court a virgin female in a group of mated females (Hollis and Kawecki 2014). To examine potential underlying mechanisms of this response, we also investigated changes in expression of genes that have been linked to cognition and have been shown to change in adult males kept in the presence of a rival (Mohorianu et al. 2017). We hypothesised that if cues of future sperm competition stimulated cognitive processes, then males reared at high density or in the presence of adult males would show increased adult learning ability (i.e. they would be quicker to learn the olfactory task and show a greater ability to focus courtship on virgin females) and an increase in the relative expression of the cognition-related genes compared to individuals from less social larval conditions. Conversely, if there is a trade-off between investment in neural processes or reproductive tissue, then adults that had been raised at low density or without adults present should show increased cognitive abilities. 


\section{Methods}

All flies used were Drosophila melanogaster wild type Dahomey strain as used previously in our studies (Bretman et al. 2016; Rouse and Bretman 2016; Leech et al. 2017). Flies were reared at $25^{\circ} \mathrm{C}$ in a humidified room, under a 12:12 light: dark cycle on an agar-sugaryeast diet. Adults were provided with purple grape juice agar plates on which to lay eggs, and the larvae were transferred approximately $24 \mathrm{~h}$ later to larval social treatments in plastic vials containing $7 \mathrm{ml}$ of fresh sugar-yeast medium, supplemented with live yeast paste. Allocation to larval treatment groups was haphazard. In order to test how rearing density affected cognition, larvae were kept at low (20 larvae per vial) or high (200 larvae per vial) density on a concentrated food medium to prevent this becoming limiting at high density (Bretman et al. 2016). In order to test the effect of adult male presence, larvae were reared at a standard density (100 larvae per vial) with or without 20 adult males aspirated into the vial. These adult males were collected from standard density vials approximately 5 days prior to the experiment, and had been kept in single-sex groups of 10 until this point. They were removed from the vials the day before eclosion of experimental flies. Experimental flies were collected within $8 \mathrm{~h}$ of eclosion, anaesthetized on ice and sexed. Males were kept in single sex groups of 10 until they were used in the learning assays. For body mass measurements, groups of 10 1-day-old flies were frozen at $-20{ }^{\circ} \mathrm{C}$, thawed at $25{ }^{\circ} \mathrm{C}$ for $30 \mathrm{~min}$, then their wet weight measured three times, and the mean of these used in subsequent analysis.

\section{Learning Ability in a Sexual Context}

Adult males were tested in a complex sexual context assay for their ability to find and court a virgin female contained in a group of 4 mated females at 5-6 days old, hereafter "virgin-finding assay," as described by Hollis and Kawecki (2014). Since female flies become unreceptive to male advances after mating due to the transfer of seminal fluid proteins (Wolfner 1997), males should learn which females have been mated and focus their courtship behavior on the virgin female. Virgin females were collected from separate standard vials and kept in groups of 10 . The day before the assay, these females were anaesthetized using $\mathrm{CO}_{2}$, and had the tips of their wings clipped to allow identification during the assay, as per previous studies (Bretman et al. 2012; Leech et al. 2017). This does not appear to influence the response of male flies to rivals (Bretman et al. 2009). To produce mated females, 10 adult males were aspirated into separate vials containing virgin females the day before the assay and left overnight to mate. In total, 4 mated females and 1 virgin female were aspirated into a new vial and left for $5 \mathrm{~min}$ to acclimate. One male from each larval treatment was then added to a single vial. Vials were scanned for a total of $20 \mathrm{~min}$, with each male observed once per minute for approximately $6 \mathrm{~s}$ each. The male was scored on whether he was courting, and if this courting was directed toward the correct (virgin) female. Males and virgin females were removed after each run, and mated females swapped every two runs. The order of treatment males was reversed such that the group added first during the initial run was added second to reduce the possibility for timing and mated female effects. The assay was carried out in a humidified room at $25^{\circ} \mathrm{C}$ and in total, three independent replicates, each consisting of 30 males per treatment, were carried out.

\section{Learning Ability in a Non-Sexual Context}

Flies from the different larval conditions were tested in an associative learning assay for their ability to associate an aversive shock stimulus with an odor in a T-maze (Hollis and Kawecki 2014). The assay was carried out under a lamp covered with a red lighting filter to reduce visual cues and at a temperature of $23 \pm 2{ }^{\circ} \mathrm{C}$. The odors used were 3-octanol (OCT) and 4-methylcyclohexanol $(\mathrm{MCH})$ at concentrations of $27 \mu \mathrm{l}$ and $10 \mu \mathrm{l}$ respectively diluted in $10 \mathrm{ml}$ of light mineral oil. These odors are commonly used in studies to test olfactory learning ability in D. melanogaster (Tully and Quinn 1985; Mery and Kawecki 2005; Hollis and Kawecki 2014). Odor concentrations had been balanced prior to the start of the assay to ensure one odor was not more influential on fly behavior than the other. To do this, flies were tested in groups, and the odor concentrations were considered balanced when the flies were distributed evenly between the two odors. The odors were pulled through a T-maze using a vacuum pump and the shock stimulus was administered by applying the holding tube containing the fly to a mechanical vortex mixer. Flies were initially given an odor preference test by measuring how long they spent in each arm of the T-maze over $2 \mathrm{~min}$. The preferred odor was then paired with the 
shock stimulus during the training period (ClaridgeChang et al. 2009). Training cycles consisted of $1 \mathrm{~min}$ odor exposure paired with a mechanical shock given for $1 \mathrm{~s}$ every $5 \mathrm{~s}$, followed by 30s of air only, then $1 \mathrm{~min}$ of exposure to the alternate odor without shock, followed by another 30 s of air. This was repeated twice. Flies were immediately tested for learning ability by giving a choice between both odors in the T-maze for $2 \mathrm{~min}$, and the amount of time spent in each arm (rather than the mid-section which was considered no choice) was recorded. Larval treatment groups were tested alternately, and the side of odor presentation was changed after every second fly. The odors were replenished approximately every 10 flies, and the experimenter was blind to treatment group during the assay. As learning ability increases during the first week of adulthood (Guo et al. 1996), flies were either tested at 1 day old or at a later stage. For larval density flies, this was at 4-8 days old, and for adult presence flies, this was at 5 days old.

\section{RT-qPCR of Learning and Memory-Associated Genes}

Expression of genes involved in learning and memory that had been identified as differing in the presence of rival males in adult $D$. melanogaster (Mohorianu et al. 2017) were examined in 1-day-old adult males reared under different larval conditions using RT-qPCR. Genes involved in learning and memory, namely dikar which is involved in long-term memory formation (Akalal et al. 2011), and dunce which produces an enzyme involved in learning (Gervasi et al. 2010) were examined; bruchpilot, a cytoskeletal protein gene involved in the release of neurotransmitters (Kittel et al. 2006), futsch and Neurexin-1, which are both involved in the structural formation and growth of synapses (Roos et al. 2000; Zeng et al. 2007) were also investigated. Primers were designed with a melting temperature of $60 \pm 1{ }^{\circ} \mathrm{C}$, and a CG content of $20-80 \%$, with an efficiency between 90 and $110 \%$ in dilution series and pipetting accuracy greater than 0.99 . Flies were snap frozen in liquid nitrogen, and heads were removed. Each sample consisted of approximately 50 heads per treatment group. These were stored at $-80^{\circ} \mathrm{C}$. Seven independent replicates for each group were collected. RNA was extracted from the samples by grinding the heads using a micropestle and using Direct-zol ${ }^{\mathrm{TM}}$ RNA miniprep kit as per the manufacturer's protocol. The RNA was checked on a Nanodrop for purity and $1 \%$ agarose gel to check for degradation. This was then converted to
cDNA using the First Strand cDNA synthesis kit (Thermo Fisher) following the manufacturer's protocol. A negative control containing no reverse transcriptase was also produced. Relative quantities were calculated by subtracting the $\mathrm{Cq}$ of each sample from that of the same reference sample and raising the primer efficiency to this number. Normalized expression for each gene was calculated by dividing the relative quantity by the geometric mean of two housekeeper genes. These were Ef1 (eukaryotic translation elongation factor 1) and Rap 21 (Ras-associated protein 2-like), and had previously been identified as candidate reference genes (Ling and Salvaterra 2011).

\section{Statistical Analyses}

Analysis was conducted using R v.3.3.3 (R Core Team 2013) and SPSS v21. For Generalised Linear Models (GLM) or Generalised Linear Mixed Models (GLMMs) using the package lme4, the maximal model was simplified using Analysis of Deviance (AOD) to assess the effect of removing terms. For the virgin-finding assay, the number of males courting and, of those courting males, the number of males courting the virgin female, were totalled per minute for each repeat. Individuals that mated during the assay were removed from the remainder of the analysis so that these numbers were calculated from the numbers available for courting. Flies were scored as courting or not courting, and courting correct or incorrect female, therefore GLMMs with binomial error distribution were used, with larval social treatment and time as fixed factors, and replicate assay as a random effect. An interaction between social treatment and time would suggest a change in courtship speed of males from one of the treatments. The overall correct courting for each group was also analysed with Mann Whitney U tests.

For the associative learning assay, flies that did not make a choice within the 2 min were excluded from the analysis. Whether the naïve flies showed a pre-training preference for either odor was tested using a GLM with a Gamma error structure, with age and social treatment as factors predicting time spent in the unconditioned odor. We also tested whether then there was a bias in which was used as the aversive odor using Chi-square tests. Post-training, we classified flies as having learnt if they spent any time in the unconditioned (i.e. previously non-preferred) odor, and tested whether there was a difference in number of learners based on age or social 
treatment using Chi-square tests. We also tested whether the proportion of time spent in the unconditioned odor was altered using GLMMs with binomial errors and logit link function. Social treatment and age were fixed factors, time spent during the pre-training in the unconditioned odor as a covariate and initial odor preference $(\mathrm{OCT}$ or $\mathrm{MCH})$ as a random effect.

Log normalized gene expression was analysed using a GLMM with gene identity and social environment as fixed effects, and biological replicate as a random factor. Where an effect of social environment was found, expression for each gene separately was analysed with post hoc tests using lsmeans and corrected for multiple testing using the Bonferroni method.

\section{Results}

\section{Effect of Larval Density}

Larval density had no effect on adult body mass (low density mean \pm S.E.M. $=0.94 \mathrm{mg} \pm 0.008$, high density $=0.93 \mathrm{mg} \pm 0.01 ; \quad \mathrm{MWU}=1744.5$; $n=60 ; p=0.892$ ). For the virgin-finding assay, there was no significant interaction between time and larval density for the proportion of males courting females (Analysis of Deviance $\chi^{2}=0.628 ; \mathrm{df}=1,5 ; p=0.428$; Fig.1a). Larval density did not affect the proportion of males courting (AOD $\chi^{2}=2.508 ; \mathrm{df}=1,4 ; p=0.113$ ), but the proportion of males courting increased over the assay period (AOD $\chi^{2}=162.220 ; \mathrm{df}=1,3 ; p<0.001$ ). There was no interaction between time and larval density for the proportion of males courting the virgin female (AOD $\chi^{2}=0.001 ; \mathrm{df}=1,5 ; p=0.973$; Fig. 1b), but there was a general increase in correct courting over time (AOD $\left.\chi^{2}=13.877 ; \mathrm{df}=1,4 ; \mathrm{p}<0.001\right)$. Larval density had a significant effect on correct courting (AOD $\chi^{2}=4.853 ; \mathrm{df}=1,4 ; p=0.028$ ), with a larger percentage of males from low density vials correctly courting, particularly during the middle period of the assay. Combining across the whole observation period showed males raised at low density spent more time courting the virgin female $(U=1388$, $N=60, p=0.030$; Fig. 1c).

For the associative learning T-maze assay, before training there was no effect of density or age on the proportion of time in the subsequently unconditioned odor (density $\mathrm{x}$ age $\chi^{2}=0.010, \mathrm{df}=1, p=0.226$; age $\chi^{2}=0.023 ; \mathrm{df}=1 ; p=0.065 ;$ density $\chi^{2}=0.002, \mathrm{df}=1$,
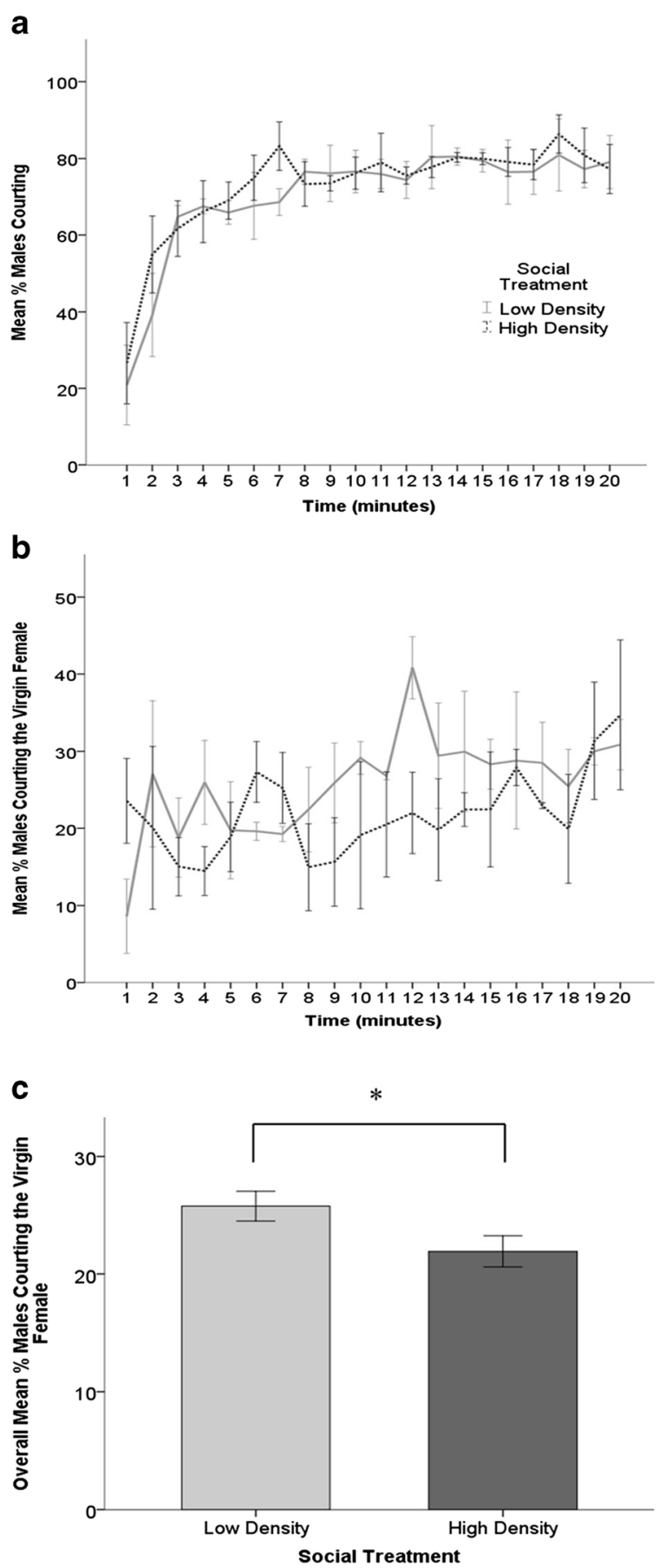

Fig. 1 Effect of larval density on male ability to correctly court virgin females. The mean percentage of three independent assays of 30 males of (a) males courting any female and (b) males courting the virgin female at each minute following male introduction, and (c) overall mean \% males courting the virgin female. Males were reared as larvae at high or low density and tested at 5-6 days posteclosion. Error bars represent $1 \pm$ S.E. * denotes significant differences $(p<0.05)$ 
$145 ; p=0.552)$. There was no significant difference in the number of flies selecting each odor pre-training between larval densities at 1 day old $\left(\chi^{2}=0.070 ; \mathrm{df}=\right.$ $1 ; p=0.792)$ or $4-8$ days old $\left(\chi^{2}=1.377 ; \mathrm{df}=1 ; p=\right.$ $0.241)$. This suggests that there were no inherent sensory biases or preferences for the odors with respect to age and social treatment. After training, the proportion of flies that had learnt was not affected by either density or age. At 1 day old $46 \%$ of low density and $43 \%$ of high density flies had learnt $\left(\chi^{2}=0.820 ; \mathrm{df}=1 ; p=0.365\right)$, for 4-8 day old flies this was $63 \%$ of low density flies and $47 \%$ of high density flies $\left(\chi^{2}=1.270 ; \mathrm{df}=1 ; p=\right.$ $0.171)$. The proportion of time spent in the unconditioned odor was not affected by density (AOD $\chi^{2}=$ 0.545; $\mathrm{df}=1 ; p=0.461$ ), but there was a significant effect of an interaction between age and pre-training preference (AOD $\chi^{2}=4.874 ; \mathrm{df}=1 ; p=0.027$ ). Younger flies that showed a stronger pre-training preference subsequently spent less time in the unconditioned odor, but this pattern was reversed in older flies. Hence larval density did not affect adult learning ability in this simple odor-association assay.

Using normalized gene expression across all genes of interest, there is no interaction between gene identity and social environment (Analysis of Deviance: $\chi^{2}=$ 6.705; df $=4,12 ; p=0.152)$, but significant effects of gene identity $\left(\chi^{2}=26.051 ; \mathrm{df}=4,8 ; p<0.001\right)$ and of larval density $\left(\chi^{2}=14.982 ; \mathrm{df}=1,8 ; \mathrm{p}<0.001\right)$, with a general pattern of relatively higher expression in low density flies (Fig. 2). Comparisons within genes using post hoc tests corrected for multiple testing, revealed only a significant increase in expression in Neurexin-1 and futsch in low density flies (bruchpilot $p=0.436$; dikar $p=0.108$; dunce $p=0.660$; futsch $p=0.005$; Neurexin-1 $p=0.003$ ).

\section{Effect of Adult Presence}

The presence of adult males during the larval stage had no effect on adult body mass (without adults present mean \pm S.E. $\mathrm{M} .=0.91 \mathrm{mg} \pm 0.008 \mathrm{~g}$, with adults present $=0.90 \mathrm{mg} \pm 0.008 ; \mathrm{t}=1.008 ; \mathrm{df}=118 ; p=0.315$ ). There was no interaction between time and adult presence on the proportion of males courting females in the virgin-finding assay ( $\mathrm{AOD} \chi^{2}=0.022 ; \mathrm{df}=1,5 ; p=$ 0.881 ; Fig. $3 a$ ) or for the proportion of males courting the virgin female (AOD $\chi^{2}=0.133 ; \mathrm{df}=1,5 ; p=0.716$; Fig. $3 b$ ). The presence of adults during the larval stages did not affect the number of males courting (AOD $\chi^{2}=$

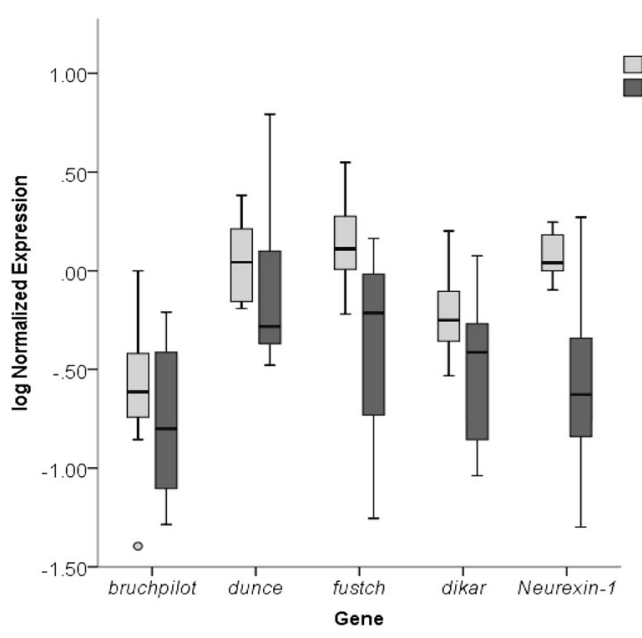

Fig. 2 Log Normalized Expression for genes in the heads of adult male flies reared at low or high larval density. Flies were 1 day old post-eclosion when frozen, and the genes examined were bruchpilot; dikar; dunce; futsch and Neurexin-1. Extreme outliers (more than 1.5x Interquartile Range) were removed

$0.388 ; \mathrm{df}=1,4 ; p=0.561$; Fig. $3 \mathrm{a})$ or the number of males correctly courting the virgin female (AOD $\chi^{2}=$ $0.681 ; \mathrm{df}=1,4 ; p=0.409$; Fig. 3b). However, there was a significant effect of time, as the number of males courting (AOD $\chi^{2}=191.46 ; \mathrm{df}=1,3 ; \mathrm{p}<0.001$; Fig. 3a) and correct courting (AOD $\chi^{2}=11.534$; $\mathrm{df}=1,3 ; \mathrm{p}<0.001$; Fig. $3 \mathrm{~b})$ significantly increased over the assay period. Overall correct courting was not significantly different between the groups $(\mathrm{U}=1570$, $N=60, p=0.227$; Fig. $3 \mathrm{c}$ ).

For the associative learning T-maze assay, before training there was no effect of adult presence or age on the proportion of time in the subsequently unconditioned odor (adult presence $\mathrm{x}$ age $\chi^{2}=0.00003, \mathrm{df}=1$, $p=0.582$; age $\chi^{2}=0.019 ; \mathrm{df}=1 ; p=0.080$; adult presence $\chi^{2}=0.002, \mathrm{df}=1,145 ; p=0.552$ ). There was no significant difference in the number of flies selecting each odor pre-training between adult presence at 1 day old $\left(\chi^{2}=0.070 ; \mathrm{df}=1 ; p=0.792\right)$ or 5 days old $\left(\chi^{2}=\right.$ $1.377 ; \mathrm{df}=1 ; p=0.241)$. Again, this suggests that there were no inherent sensory biases or preferences for the odors with respect to age and social treatment. After training, the percentage of flies that had learnt was not affected by either adult presence or age. At 1 day old, $61 \%$ of adult absence and $68 \%$ of adult presence flies had learnt $\left(\chi^{2}=0.156 ; \mathrm{df}=1 ; p=0.693\right)$; for 5 day old flies this was $68 \%$ for both treatments. The proportion of time spent in the unconditioned odor was not affected by 
a

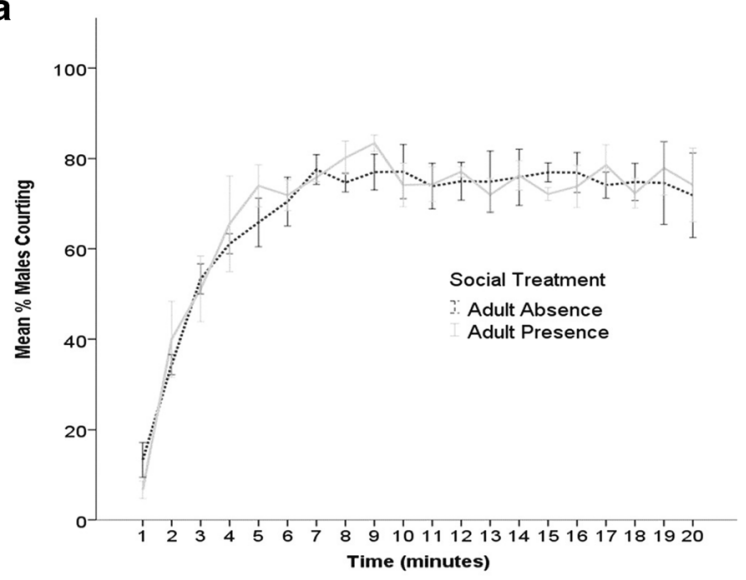

b
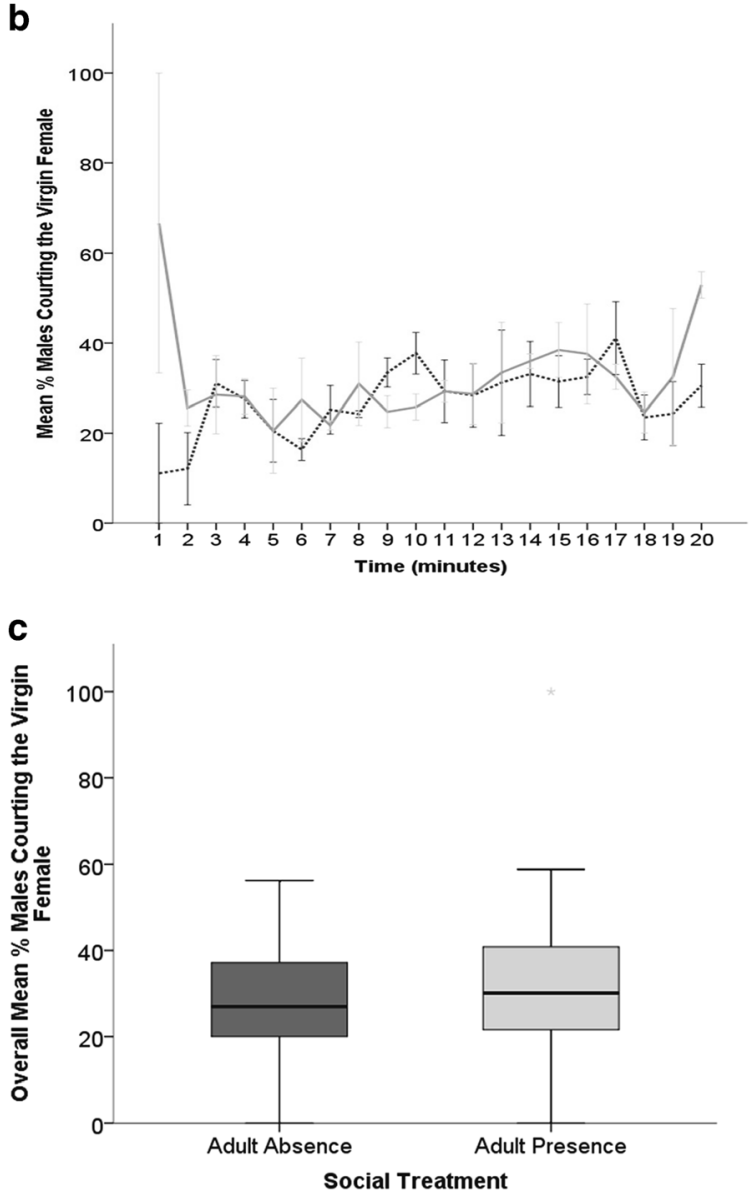

Fig. 3 Effect of adult presence during larval stage on adult male ability to correctly court virgin females. The mean percentage of three independent assays of 30 males of (a) males courting any female and (b) males courting the virgin female at each minute following male introduction, and (c) overall mean \% males courting the virgin female. Males were reared as larvae with or without adult males present, and tested at 5-6 days post-eclosion. Error bars represent $1 \pm \mathrm{S}$.E adult presence $\left(\mathrm{AOD} \chi^{2}=1.067 ; \mathrm{df}=1,4 ; p=302\right)$, age (AOD $\chi^{2}=0.867 ; \mathrm{df}=1,4 ; p=0.352$ ) or pre-training time spent in the unconditioned odor (AOD $\chi^{2}=$ $0.146 ; \mathrm{df}=1,4 ; p=0.703$ ).

Using normalized gene expression across all genes of interest (Fig. 4), there is no interaction between gene identity and social environment $\left(\chi^{2}=0.667 ; \mathrm{df}=4,12\right.$; $p=0.955)$, but differences in expression between genes $\left(\chi^{2}=1.998, \mathrm{df}=4,8, p=0.027\right)$, and no effect of adult presence $\left(\chi^{2}=2.777\right.$, df $\left.=1,8, p=0.096\right)$. Post hoc analysis for within gene effects likewise showed there was no effect of adult presence on normalized expression (bruchpilot $p=0.545$; dikar $p=0.347$; dunce $p=$ 0.789; futsch $p=0.234$; Neurexin-1 $p=0.632$; Fig. 4). Therefore we find no evidence for the presence of adults affecting learning in either behavioral assay or on the expression of neuronal genes.

\section{Discussion}

These results suggest that larval social conditions can affect adult learning in male $D$. melanogaster. However, this was dependent on both the type of larval conditions and the learning assay used. Contrary to our predictions that high larval density and the presence of adults during larval stages would increase overall learning ability in young adult males through increased social stimulation, we observed that males from low larval densities had

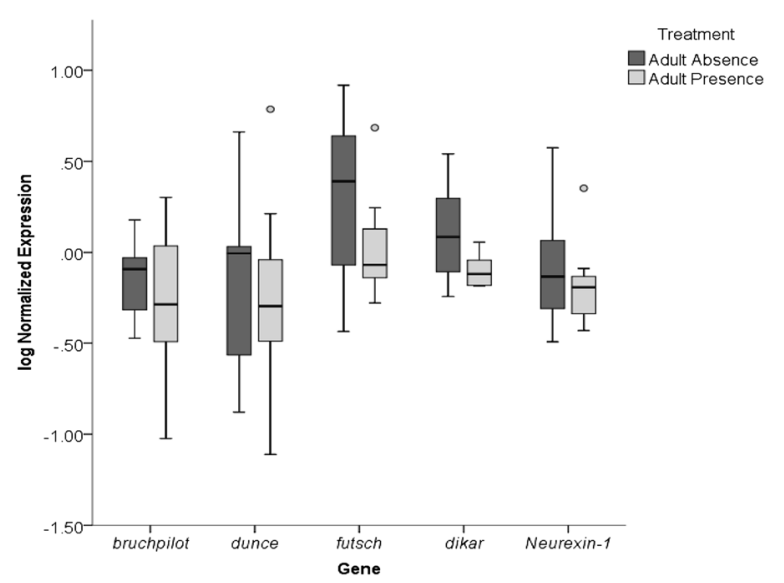

Fig. 4 Log Normalized Expression for genes in the heads of adult male flies reared with or without adult males in the larval environment. Flies were 1 day old post-eclosion when frozen, and the genes examined were bruchpilot; dikar; dunce; futsch and Neurexin-1. Extreme outliers (more than 1.5x Interquartile Range) were removed 
higher learning ability in the virgin-finding assay compared to those from high densities. This was not simply due to a difference in activity, as density did not affect the amount of courting per se. However, there was no significant difference in an associative learning assay. There was a general trend for increased expression of learning and memory-associated genes in males from low densities, but when genes were analysed individually this was only significant for futsch and Neurexin-1. The presence of adult males in the larval environment had no significant effect on learning ability of adult males in either of the learning assays used and no effect on expression of any of the genes examined.

\section{Larval Density Affects Adult Learning}

We had expected that high density males would show an increased learning ability due to the provision of cues that indicate future sperm competition stimulating cognitive processes, and the greater requirement for processing of these cues. Despite high density males increasing accessory gland size in response to these larval conditions (Bretman et al. 2016), these individuals had poorer overall correct courting scores in the virgin finding assay than those from low larval densities. Therefore, it is possible that adult flies kept at high density as larvae are allocating resources to factors other than cognition, such as increased accessory gland size. For example, bees, Apis mellifera, that are immunechallenged have decreased ability to learn an association than those that have not been challenged (Mallon et al. 2003). Moreover, formation of long-term memory in fruit flies results in decreased stress tolerance (Mery and Kawecki 2005), and fly lines selected for learning ability have reduced lifespans (Burger et al. 2008). Previously, Mery and Kawecki (2004) have found a reproductive cost of learning, measured as egg laying rate, in individuals subjected to conditioning from lines selected for high learning under poor nutritional conditions compared to those not subjected to the conditioning. Therefore, it is possible that investment in reproduction, such as the production of larger accessory glands, could represent a trade-off against cognition in high density flies. Evidence for trade-offs between reproduction and cognition have been found in, for example, butterflies, Pieris rapae, (Snell-Rood et al. 2011), and bats (Pitnick et al. 2006). Therefore, it is possible that the production of larger accessory glands in high density males diverts resources to the production of this reproductive trait, and decreases the availability of energy for cognition. A trade-off such as this may be expected to be particularly significant under stressful conditions, for example, fly lines selected for adaptation to poor quality food sources show a reduced learning ability (Kolss and Kawecki 2008). Further, an absence of sexual selection in fruit flies results in a decreased learning ability (Hollis and Kawecki 2014). As cognition is important for males to identify and assess both mating partners and potential rivals, an increased investment in cognition would be beneficial under such social conditions. However, where there is a requirement for increased reproductive tissue, for example, in the presence of rival males, this could necessitate investment into reproductive tissue. Therefore increased mating competition might select for increased investment in various traits and tissues, and how resources are differentially directed into each requires further investigation.

Another possible explanation for changed cognitive output is that, even in the presence of excess food, larvae at high density could interfere with the feeding of others. This could, for example, increase stress or reduce food consumption (Goss-Custard 2002), resulting in an overall reduction in available energy and coupled with increased investment in accessory gland growth, decrease the energy for cognitive investment. However, it is worth noting that our manipulations did not alter adult body mass, suggesting that food consumption was not limited. Additionally, for populations reared at high larval densities faster development times have been connected to both feeding rates and tolerance to waste build-up (Joshi and Mueller 1988; Mueller et al. 1991; Joshi 2001). There may also be an effect on the microbial community on the food which could alter the microbiome of flies between treatments. It is therefore possible that an increased build-up of waste products at high densities could impact cognitive development in some manner.

However, it seems likely that high density larvae are in contact with one another more or less constantly, but the low density individuals may not be. The variability in the frequency of contact for larvae kept at low densities would create inconsistent, variable social cues and may result in an increased requirement for neural plasticity (Fawcett and Frankenhuis 2015). Intriguingly, low density flies had non-significant higher average learning indices in the associative learning task than high density flies, which was a consistent trend over the assay sessions. However, the virgin-finding assay is more likely 
to represent a natural situation than the associative learning task, and it may be argued, therefore, that it is the more relevant assay for this reason. However, instead of rather than a difference in learning ability per se, the differences observed between low and high larval density flies could be a result of a difference in sensory perception. Fruit flies are known to use multiple cues in the detection of rivals (Bretman et al. 2011; Maguire et al. 2015), and social experience in adult flies can affect behavior in male-male interactions, which is at least partly mediated by perception of pheromones (Svetec and Ferveur 2005). Indeed, courtship conditioning responses, whereby experience with a mated female alters male behavior towards a virgin female (Siegel and Hall 1979), is known to be largely dependent on detection of the pheromone cis-vaccenyl acetate (cVA) (Ejima et al. 2007). Thus, it is possible that a difference in sensory ability to perceive cues, such as cVA could be having an influence in the learning assays, and it would be interesting to explore this possibility in more detail, including utilising flies with defective sensory structures.

We analysed expression of genes involved in a number of aspects of learning, memory and synaptic plasticity that had previously been shown to socially responsive in adult flies (Mohorianu, Bretman et al. 2017). We found a general pattern of increased relative expression in flies raised at low density. In particular, futsch and Neurexin-1, which are both involved in synapse formation (Roos et al. 2000; Zeng et al. 2007), showed an increased expression in low density flies. An enhancement in synaptic plasticity through expression changes in these genes could underpin the enhanced ability of low density flies in the virgin-finding assay. Futsch is involved in the growth of synapses by the regulation of microtubule cytoskeleton organisation (Roos et al. 2000), and mutants display neurodegeneration and reduced learning abilities (Bettencourt da Cruz et al. 2005). Neurexin-1 expression is associated with sleep (Larkin et al. 2015), a socially responsive behavior that has been linked to synaptic plasticity in adults (Ganguly-Fitzgerald et al. 2006; Donlea et al. 2014). Taken with our work this suggests that synaptic plasticity mediated by Neurexin- 1 could be affected by changes in social conditions across life stages in fruit flies. However, it should be noted that there could be other explanations for the pattern of higher relative expression from low density males. For example, larval crowding increases lipid stores in the bodies of adult flies (Borash and Ho 2001). We have not determined whether this is the case for our manipulations, though we do not find that they alter body mass overall. Nevertheless, an increased lipid store in the heads of the males from high densities could potentially influence the proportion of mRNA that is specifically taken from the brain, although the potential contribution of this is currently undetermined.

Adult Presence during Larval Stages Does Not Affect Adult Learning Ability

There was no significant effect of adult presence on learning ability for either of the assays used here or on the expression of any of the genes examined. We had expected that these individuals would have an increased learning ability due to the processing of cues that indicate future reproductive competition and results in increased accessory gland size of these males, or, instead, that a trade-off between these responses would occur. Possibly in contrast to the differences observed in the density groups, there does not seem to be a trade-off between the production of larger accessory glands and cognition. Similarly to the high density group, however, adult male presence may provide a consistent, stable level of information provision that does not require an increase in synaptic plasticity (Fawcett and Frankenhuis 2015). These results may be unsurprising as another study found no effect of rearing larvae in complex feeding environments on adult learning ability (Durisko and Dukas 2013). There was, however, an extremely consistent trend for non-significant higher learning scores across assay sessions in the associative learning task for the adult presence males.

Adult $D$. melanogaster increase their learning ability during the first week of adulthood (Guo et al. 1996). The associative learning task was carried out at 1 day as we envisaged that any difference would be more likely to be detectable at this stage, but was also tested later in the first week when learning ability is expected to have increased (Guo et al. 1996). However, there was no difference at either age examined in density or adult presence groups. Changes in environmental conditions that result in a mismatch of phenotype to environment could be costly (DeWitt et al. 1998). Further, as neural structures are energetically expensive to produce (Niven and Laughlin 2008), and as D. melanogaster brains continue to show plasticity into adulthood (Donlea et al. 2014), it may be more beneficial, if there are 
changes in cognition in response to reproductive cues, that this is largely affected during the adult stage. As the social environment can be variable, plasticity at sexual maturity could represent a route that is less likely to result in mismatches for males responding to these types of cues, and may be particularly important at these stages.

Due to the differences in experimental setup, we cannot directly compare between the density and adult presence social environments, but taking both sets of results into consideration, it appears that the type of cues available may have distinct effects. As group burrowing appears to require both visual and mechanosensory cues (Dombrovski et al. 2017), these cues may be important for the larvae, and as our learning paradigms both largely involve olfactory learning, it may be speculated that differences in other aspects of cognition, for example, visual learning, which would not be specifically identified in these assays, could vary. Additionally, despite both high density and adult presence males showing the same phenotype of increased accessory gland growth, there does appear to be differences between these groups. If the presence of individuals of a similar age represents a more accurate predictor of future sperm competition than the presence of individuals from an earlier cohort, this may affect investment into cognition to process these cues. Larvae of the same age are more likely to be direct reproductive competitors, so investing more to process cues under these circumstances could be beneficial. Alternatively, these larval social conditions may be differentially stressful, and this could impact on cognition. For example, early life stress in rats can influence neurogenesis (Mirescu et al. 2004), and the formation of long-term memory has been found to decrease stress tolerance in D. melanogaster (Mery and Kawecki 2005).

In conclusion, we have found evidence that larval social conditions can affect adult male learning ability in Drosophila melanogaster. Flies from low density larval environments performed better in a learning assay than those from high density conditions. We found an overall effect on gene expression, suggesting larval density could affect synaptic plasticity. It is unclear whether this represents enhanced responses to variable cues in low density males, or reduced cognition in high density males, perhaps through a reproduction-cognition tradeoff. The presence of adult males in the larval environment had no significant effect on learning ability of adult males. These results suggest that the type of cues available may have distinct effects on the development of certain aspects of cognition. Processing information about the sperm competition environment and responding appropriately can have important fitness effects (Bretman et al. 2009; Wigby et al. 2009), and it appears that the social conditions experienced during early life stages could have potential implications for the cognitive abilities of these individuals into adulthood. Whether cues of sperm competition received during adulthood also influence cognitive abilities remains to be tested.

Acknowledgements We thank Thomas Leech for help with experimental work, Elizabeth Duncan for advice on gene expression analysis and two anonymous reviewers for their helpful comments on the manuscript.

Funding LM was supported by the Boothman, Reynolds and Smithells scholarship at the University of Leeds, JR by a Leverhulme Trust grant RPG-2016-184 to AB and Elizabeth Duncan, and AB by a University of Leeds Academic Fellowship.

Data Availability Data is archived in the open access Leeds Data Repository https://doi.org/10.5518/580

\section{Compliance with Ethical Standards}

Conflict of Interest The authors declare that they have no conflict of interest.

Open Access This article is distributed under the terms of the Creative Commons Attribution 4.0 International License (http:// creativecommons.org/licenses/by/4.0/), which permits unrestricted use, distribution, and reproduction in any medium, provided you give appropriate credit to the original author(s) and the source, provide a link to the Creative Commons license, and indicate if changes were made.

\section{References}

Akalal D-BG, Yu D, Davis RL (2011) The long-term memory trace formed in the Drosophila $\alpha / \beta$ mushroom body neurons is abolished in long-term memory mutants. J Neurosci 31 : 5643-5647

Bettencourt da Cruz A, Schwärzel M, Schulze S, Niyyati M, Heisenberg M, Heisenberg D (2005) Disruption of the MAP1B-related protein FUTSCH leads to changes in the neuronal cytoskeleton, axonal transport defects, and progressive neurodegeneration in Drosophila. Mol Cell Biol 16: 2433-2442

Borash DJ, Ho GT (2001) Patterns of selection: stress resistance and energy storage in density-dependent populations of Drosophila melanogaster. J Insect Physiol 47:1349-1356 
Bretman A, Fricke C, Chapman T (2009) Plastic responses of male Drosophila melanogaster to the level of sperm competition increase male reproductive fitness. P R Soc B 276:1705-1711

Bretman A, Westmancoat JD, Gage MJG, Chapman T (2011) Males use multiple, redundant cues to detect mating rivals. Curr Biol 21:617-622

Bretman A, Westmancoat JD, Gage MJG, Chapman T (2012) Individual plastic responses by males to rivals reveal mismatches between behavior and fitness outcomes. P R Soc B 279:2868-2876

Bretman A, Fricke C, Westmancoat JD, Chapman T (2016) Effect of competitive cues on reproductive morphology and behavioral plasticity in male fruitflies. Behav Ecol 27:452-461

Bretman A, Rouse J, Westmancoat JD, Chapman T (2017) The role of species-specific sensory cues in male responses to mating rivals in Drosophila melanogaster fruitflies. Ecol Evol 7:9247-9256

Burger JMS, Kolss M, Pont J, Kawecki TJ (2008) Learning ability and longevity: a symmetrical evolutionary trade-off in Drosophila. Evolution 62:1294-1304

Claridge-Chang A, Roorda RD, Vrontou E, Sjulson L, Li H, Hirsh J, Miesenböck G (2009) Writing memories with lightaddressable reinforcement circuitry. Cell 139:405-415

DeWitt TJ, Sih A, Wilson DS (1998) Costs and limits of phenotypic plasticity. Trends Ecol Evol 13:77-81

Dombrovski M, Poussard L, Moalem K, Kmecova L, Hogan N, Schott E, Vaccari A, Acton S, Condron B (2017) Cooperative behavior emerges among Drosophila larvae. Curr Biol 27: 2821-2826

Donlea JM, Ramanan N, Silverman N, Shaw PJ (2014) Genetic rescue of functional senescence in synaptic and behavioral plasticity. Sleep 37:1427-1437

Dukas R (2008) Evolutionary biology of insect learning. Annu Rev Entomol 53:145-160

Durisko Z, Dukas R (2013) Effects of early-life experience on learning ability in fruit flies. Ethology 119:1067-1076

Ejima A, BPC S, Lucas C, Van Der Goes Van Naters W, Miller CJ, Carlson JR, Levine JD, Griffith LC (2007) Generalization of courtship learning in Drosophila is mediated by cis-vaccenyl acetate. Curr Biol 17:599-605

Fawcett TW, Frankenhuis WE (2015) Adaptive explanations for sensitive windows in development. Front Zool 12:S3-S3

Ganguly-Fitzgerald I, Donlea J, Shaw PJ (2006) Waking experience affects sleep need in Drosophila. Science 313: 1775-1781

Gervasi N, Tchénio P, Preat T (2010) PKA dynamics in a Drosophila learning center: coincidence detection by rutabaga adenylyl cyclase and spatial regulation by dunce phosphodiesterase. Neuron 65:516-529

Goss-Custard JD (2002) Competition for food and interference among waders. Ardea 38-90:31-52

Guo A, Li L, Xia S, Feng C, Wolf R, Heisenberg M (1996) Conditioned visual flight orientation in Drosophila: dependence on age, practice, and diet. Learn Mem 3:49-59

Heisenberg M, Heusipp M, Wanke C (1995) Structural plasticity in the Drosophila brain. J Neurosci 15:1951-1960

Hollis B, Kawecki TJ (2014) Male cognitive performance declines in the absence of sexual selection. P R Soc B 281:20132873

Joshi A (2001) Development and competition in Drosophila: a tale of two densities. P Indian Nat Sci Acad B 67:389-396
Joshi A, Mueller LD (1988) Evolution of higher feeding rate in Drosophila due to density-dependent natural selection. Evolution 42:1090-1093

Kasumovic MM, Bruce MJ, Andrade MCB, Herberstein ME (2008) Spatial and temporal demographic variation drives within-season fluctuations in sexual selection. Evolution 62: 2316-2325

Kittel RJ, Wichmann C, Rasse TM, Fouquet W, Schmidt M, Schmid A, Wagh DA, Pawlu C, Kellner RR, Willig KI, Hell SW, Buchner E, Heckmann M, Sigrist SJ (2006) Bruchpilot promotes active zone assembly, $\mathrm{Ca} 2+$ channel clustering, and vesicle release. Science 312:1051-1054

Kolss M, Kawecki TJ (2008) Reduced learning ability as a consequence of evolutionary adaptation to nutritional stress in Drosophila melanogaster. Ecol Entomol 33:583-588

Larkin A, Chen M-Y, Kirszenblat L, Reinhard J, van Swinderen B, Claudianos C (2015) Neurexin-1 regulates sleep and synaptic plasticity in Drosophila melanogaster. Eur J Neurosci 42: 2455-2466

Leech T, Sait SM, Bretman A (2017) Sex-specific effects of social isolation on ageing in Drosophila melanogaster. J Insect Physiol 102:12-17

Lévy F, Melo AI, Galef BG, Madden M, Fleming AS (2003) Complete maternal deprivation affects social, but not spatial, learning in adult rats. Dev Psychobiol 43:177-191

Liedtke J, Schneider JM (2017) Social makes smart: rearing conditions affect learning and social behavior in jumping spiders. Anim Cogn 20:1093-1106

Ling D, Salvaterra PM (2011) Robust RT-qPCR data normalization: validation and selection of internal reference genes during post-experimental data analysis. PLoS One 6:e17762

Maguire CP, Lizé A, Price TAR (2015) Assessment of rival males through the use of multiple sensory cues in the fruitfly Drosophila pseudoobscura. PLoS One 10:e0123058

Maleszka J, Barron AB, Helliwell PG, Maleszka R (2009) Effect of age, behavior and social environment on honey bee brain plasticity. J Comp Physiol A 195:733-740

Mallon EB, Brockmann A, Schmid-Hempel P (2003) Immune response inhibits associative learning in insects. P R Soc B 270:2471-2473

Mery F, Kawecki TJ (2004) An operating cost of learning in Drosophila melanogaster. Anim Behav 68:589-598

Mery F, Kawecki TJ (2005) A cost of long-term memory in Drosophila. Science 308:1148-1148

Mirescu C, Peters JD, Gould E (2004) Early life experience alters response of adult neurogenesis to stress. Nat Neurosci 7:841

Mohorianu I, Bretman A, Smith DT, Fowler EK, Dalmay T, Chapman T (2017) Genomic responses to the socio-sexual environment in male Drosophila melanogaster exposed to conspecific rivals. RNA 23:1048-1059

Mueller L, Guo P, Ayala F (1991) Density-dependent natural selection and trade-offs in life history traits. Science 253: 433-435

Niven JE, Laughlin SB (2008) Energy limitation as a selective pressure on the evolution of sensory systems. J Exp Biol 211: $1792-1804$

Parker GA (1970) Sperm competition and its evolutionary consequences in the insects. Biol Rev 45:525-567

Parker GA, Pizzari T (2010) Sperm competition and ejaculate economics. Biol Rev 85:897-934 
Pitnick S, Jones KE, Wilkinson GS (2006) Mating system and brain size in bats. P R Soc B 273:719-724

Prounis GS, Foley L, Rehman A, Ophir AG (2015) Perinatal and juvenile social environments interact to shape cognitive behavior and neural phenotype in prairie voles. P R Soc B 282: 20152236

R Core Team (2013) R: A language and environment for statistical computing. R Foundation for Statistical Computing, Vienna, Austria. http://www.R-project.org/

Roos J, Hummel T, Ng N, Klämbt C, Davis GW (2000) Drosophila futsch regulates synaptic microtubule organization and is necessary for synaptic growth. Neuron 26:371-382

Rouse J, Bretman A (2016) Exposure time to rivals and sensory cues affect how quickly males respond to changes in sperm competition threat. Anim Behav 122:1-8

Rouse J, Watkinson K, Bretman A (2018) Flexible memory controls sperm competition responses in male Drosophila melanogaster. P R Soc B 285:20180619

Shifferman EM (2012) It's all in your head: the role of quantity estimation in sperm competition. P R Soc B 279:833-840

Siegel RW, Hall JC (1979) Conditioned responses in courtship behavior of normal and mutant Drosophila. P Natl Acad Sci USA 76:3430-3434

Snell-Rood EC, Davidowitz G, Papaj DR (2011) Reproductive tradeoffs of learning in a butterfly. Behav Ecol 22:291-302

Sol D (2009) Revisiting the cognitive buffer hypothesis for the evolution of large brains. Biol Lett 5:130-133
Svetec N, Ferveur J-F (2005) Social experience and pheromonal perception can change male-male interactions in Drosophila melanogaster. J Exp Biol 208:891-898

Tully T, Quinn WG (1985) Classical conditioning and retention in normal and mutant Drosophila melanogaster. J Comp Physiol A 157:263-277

Wedell N, Gage JG, Parker GA (2002) Sperm competition, male prudence and sperm-limited females. Trends Ecol Evol 17: 313-320

Wigby S, Sirot LK, Linklater JR, Buehner N, Calboli FCF, Bretman A, Wolfner MF, Chapman T (2009) Seminal fluid protein allocation and male reproductive success. Curr Biol 19:751-757

Wolfner MF (1997) Tokens of love: functions and regulation of Drosophila male accessory gland products. Insect Biochem Mol Biol 27:179-192

Zeng X, Sun M, Liu L, Chen F, Wei L, Xie W (2007) Neurexin-1 is required for synapse formation and larvae associative learning in Drosophila. FEBS Lett 581:2509-2516

Publisher's Note Springer Nature remains neutral with regard to jurisdictional claims in published maps and institutional affiliations. 